\title{
Philosophiques
}

\section{Réduction et constitution dans la phénoménologie du dernier Husserl}

\section{Dan Zahavi}

Volume 20, numéro 2, automne 1993

Perspectives sur la phénoménologie et l'intentionnalité

URI : https://id.erudit.org/iderudit/027231ar

DOI : https://doi.org/10.7202/027231ar

Aller au sommaire du numéro

Éditeur(s)

Société de philosophie du Québec

ISSN

0316-2923 (imprimé)

1492-1391 (numérique)

Découvrir la revue

Citer cet article

Zahavi, D. (1993). Réduction et constitution dans la phénoménologie du dernier Husserl. Philosophiques, 20(2), 363-381. https://doi.org/10.7202/027231ar d'utilisation que vous pouvez consulter en ligne.

https://apropos.erudit.org/fr/usagers/politique-dutilisation/ 
Philosophiques, vol. XX, numéro 2, Automne 1993, p. 363-381

\title{
RÉDUCTION ET CONSIIIUTION DANS LA PHÉNOMÉNOLOGIE DU DERNIER HUSSERL*
}

\author{
par \\ Dan Zahavi
}

Je me propose ici d'approcher un aspect du concept transcendantal de constitution chez Husserl. Je m'intéresse à sa position dans la controverse réalisme / idéalisme qui porte directement sur la discussion traditionnelle concernant le statut de la constitution, soit la constitution comme restitution ou comme création ${ }^{\mathrm{I}}$.

Je vais commencer mon exposé en récapitulant quelquesunes des idées principales du projet phénoménologico-transcendantal de Husserl en insistant sur la notion de réduction. Ceci est essentiel pour une évaluation précise du but que vise

I. Afin de comprendre le concept husserlien de constitution, il serait nécessaire de différencier et analyser un groupe de problèmes connexes. I) Une distinction doit être faite entre un usage pré-transcendantal et un usage transcendantal de ce concept Lorsque Husserl parle de constitution dans les Recherches logiques, c'est dans un sens beaucoup plus restreint que dans ses écrits subséquents. 2) De plus, la distinction de Husserl entre phénoménologie statique et phénoménologie génétique nécessite aussi une distinction entre un concept statique et un concept génétique de constitution (voir par exemple Husserliana XI, p. 345). 3) A part ces deux considérations générales, il serait aussi nécessaire de faire des distinctions en ce qui concerne la description fonctionnelle du processus actuel de constitution. Juste pour mentionner quelques différences décisives ; la constitution prenant place dans l'auto-temporalisation ISelbstzeitigungl, l'expérience anté-prédicative, l'expérience catégoriale et la constitution des objets culturels sont des processus distincts, chacun demandant son propre traitement. l'analyse de ces différents aspects du concept de constitution n'est pas une tâche que je peux entreprendre dans ce texte. Voir toutefois D. Zahavi, Intentionalität und Konstitution. Eine Einführung in Husserls Logische Untersuchungen, Kopenhagen, Museum Tusculanum, 1992 ; et "Constitution and Ontology. Some Remarks on Husserl's Ontological Position in the Logical Investigations », Husserl Studies 9. 1992, p. III-I24.

- Cette étude - comprenant des éléments de ma thèse de doctorat, qui traite de la fonction constitutive de l'intersubjectivité transcendantale - est basée sur des recherches faites aux Archives Husserl à Leuven, Belgique. Je remercie le directeur des archives, le professeur $S$. Ijsseling pour la permission de citer des passages des manuscrits inédits de Husserl. 
Husserl, et je crois qu'une compréhension juste de son concept transcendantal de constitution n'est possible qu'en tenant compte de cet arrière-plan. Je présenterai ensuite brièvement la question des conséquences idéalistes des réflexions transcendantales de Husserl, et je terminerai sur un aperçu de la relation entre monde et subjectivité telle que Husserl la conçoit à partir de la fin des années vingt et qui permettra une nouvelle approche décisive pour le problème de la constitution. Ainsi, il deviendra clair que les vues de Husserl sur ces questions sont beaucoup plus en accord avec les vues des derniers phénoménologues comme Heidegger et Merleau-Ponty que ses critiques sont prêts à l'admettre.

La phénoménologie de Husserl peut être comprise comme une tentative de surmonter quelques faiblesses qui sont inhérentes à toute science mondaine - positive, objective. Selon Husserl, toutes les sciences mondaines - fonctionnant dans l'attitude naturelle - sont caractérisées par des insuffisances de principe parce qu'elles ne thématisent pas leur propre prestation intentionnelle. La conséquence de cette négligence, c'est leur incapacité de saisir le sens d'être [Seinssinnl véritable de leur propre domaine de recherche et ultimement du monde lui-même $(17 / 17 ; 5 / 160)^{2}$. Comme les sciences mondaines ne soulèvent pas ces questions concernant leur propre fondement ou bien présupposent simplement leurs réponses de façon dogmatique, elles ne possèdent pas le genre de rationalité qui est la marque distinctive d'une vraie science $^{3}$.

IDlès lors il devrait être facile de comprendre que toutes les sciences objectives manquent précisément du savoir de ce qui a plus que tout valeur de principe : il leur manque, je veux dire, le savoir de cela même qui pourrait fournir sens et validité aux formations

2. Les références à l'édition Husserliana sont données dans le texte de la façon suivante. Le premier numéro réfère au volume, le second à la page. Lorsque référant aux manuscrits inèdits, le dernier numéro réfère toujours à la page sténographiée originale.

3. Husserl reconnait toutefois que ces insuffisances sont parmi les conditions de possibilité pour le progrès continu de la science positive, et conséquemment sa critique n'entend pas imposer la nécessité d'un changement radical à l'intérieur des sciences elles-mêmes. Voir les considérations similaires de Heidegger dans Grundprobleme der Phänomenologie, Gesamtausgabe Band 24, Frankfurt, V. Klostermann, 1989, § 9 . 
théorétiques du savoir objectif en général, et du même coup leur procurer la dignité d'un savoir à partir du fondement ultime (6/i21).

La réalisation de l' idéal scientifique exige une critique radicale qui, selon Husserl, est correctement accomplie par la phénoménologie transcendantale. Une critique qui, par une élucidation simultanée de la prestation intentionnelle du sujet et du sens d'être du monde, questionne indirectement les présuppositions majeures, épistémologiques et ontologiques, des sciences mondaines (8/ $262,6 / 412$ ). Husserl décrit la tâche de la phénoménologie comme celle qui consiste à transformer l'évidence universelle de l'être du monde - la plus grande de toutes les énigmes - en quelque chose d'intelligible (6/184, 6/208), et les termes de Husserl pour les procédés phénoménologiques rendant cette tâche possible sont l'épochè et la réduction transcendantale.

Comme la totalité de la recherche phénoménologicotranscendantale de Husserl doit être comprise dans le cadre de l'épochè et de la réduction, une compréhension juste de leur fonction est d'une importance capitale pour comprendre le concept de constitution. Aussi, la plupart des disputes concernant le champ, les prétentions et la validité des analyses phénoménologiques sont basées sur différentes interprétations de l'épochè et de la réduction et ne peuvent, en conséquence, être réglées que par une clarification thematique de ces concepts.

Notre élucidation prendra place dans une confrontation explicite avec deux mésinterprétations dominantes concernant leur but. Ainsi, peu à peu, il deviendra clair que le but de Husserl - et l'objet de la phénoménologie transcendantale - n'est pas d'effectuer une annihilation du monde afin de dépeindre une subjectivité sans monde, pure et isolée, pas plus qu'elle ne doit être comprise comme une abstention d'engagements ontologiques, comme si la phénoménologie n'avait affaire qu'à une élucidation de la sphère du sens et de la signification (comme quelque chose de séparé de l'êtrel ${ }^{4}$.

4. Concernant cette demière observatiòn, elle est relièe à une interprétation spécifiquement sémantique du concept de noème. Dans cet article, je vais toutefois éviter cette question - et simplement référer à la présentation circonspecte de J.J. Drummond dans son Husserlian Intentionality and Non-Foundational Realism, Dordrecht, Kluwer Academic Publishers, rg9o, dont j'endosse l'interprétation et la critique de « l'approche frégéenne »-en essayant de démontrer comment une clarification de l'épochè et de la réduction peut en elle-mème servir de contre-exemple. 


\section{$\|$}

Nous avons vu que la tâche de la phénoménologie, selon Husserl, est de comprendre le monde (6/17i). Un prérequis nécessaire pour l'amorce de ce projet est la suspension de notre croyance naïve et dogmatique en l'existence ou la nature ontologique du monde, et ce afin d'atteindre son mode de donation [Wie ihrer Gegebenheitsweisel. Si notre investigation doit être libre de toute présupposition dogmatique concernant le statut ontologique du monde, elle doit suivre le principe des principes, à savoir de considérer toute intuition originaire comme une source légitime de connaissance et d'accepter tout ce qui s'offre à nous dans l'intuition simplement comme ce qui se présente comme étant (3/5I, 3/43). C'est dire que, dans le but d'éviter des présuppositions métaphysiques et ontologiques injustifiées, on doit entreprendre une réduction radicale vers le donné phénoménologique. Conséquemment, la mise en œuvre de l'épochè n'implique pas une exclusion [Ausschaltung] du monde, mais plutôt une suspension de notre croyance naïve en son existence. Ayant effectué l'épochè, nous ne sommes plus préoccupés aveuglément (ontiquement) par le monde ; désormais, nous nous concentrons sur son mode de donation $(8 / 502)$. En d'autres mots, nous ne nous intéressons au monde que dans la mesure où il est vécu, perçu, imaginé, jugé etc., c'est-à-dire dans la mesure où il est le corrélat d'une expérience, d'une perception, d'une imagination, etc. Ainsi, la tâche d'une découverte philosophique du monde conduit indirectement à une découverte de la subjectivité corrélative qui en fait l'expérience, étant donné que l'approche phénoménologique du monde doit nécessairement se faire par la voie de son apparaître et donc, pour la subjectivité $(8 / 263)^{5}$. Voilà une observation qui déjà nous indique la relation étroite qui existe entre ces deux sphères.

Cette approche indirecte de la subjectivité est accentuée par Husserl dans ce qu'il est convenu d'appeler la voie ontologique vers la réduction. Il décrit comment la recherche phénoménologique prend place dans des mouvements reliés et conjugués $(17 / 270)$. L'analyse d'une certaine région ontologique, considérée comme fil conducteur transcendantal [Leitfaden], nous conduit à la conscience qui en fait l'expérience ou, en termes husserliens, à la

5. À plusieurs reprises, Husserl fait remarquer que l'analyse systématique de cette corrélation est d'une importance capitale (6/169, 17/253). 
conscience constituante comme source de validite ${ }^{6}$. In concreto, ces thèmes sont inséparables $(4 / 107,6 / 175)$, mais une approche systématique doit les isoler abstraitement et les analyser un après l'autre.

Le premier point est le monde de la vie tout simplement donné, et ce avant tout tel qu'il se donne perceptivement en tant qu'existant «normal », tout simple, dans une pure certitude d'être sans faille let par conséquent hors de doute). Lorsque se fixera la nouvelle orientation de l'intérêt, et du même coup lorsqu'il sera l'objet d'une rigoureuse épochè, ce monde de la vie deviendra une première rubrique intentionnelle, l'index, le fil conducteur pour les questions en retour qui porteront sur la diversité des modes d'apparition et leurs structures intentionnelles. Une nouvelle orientation du regard, au deuxième niveau de la réflexion, conduit au pôle égologique et à ce qui est propre à son identité $(6 / 175$; voir $9 / 207)$.

Conséquemment, on doit souligner encore une fois que l'explicitation de la subjectivité constituante doit être mise en rapport avec une élucidation philosophique du monde dont elle est en fait inséparable ${ }^{7}$.

6. Dans son livre Genetische Phänomenologie und Reduktion, La Haye, Martinus Nijhoff, 1970, Aguirre soutient que tant la voie ontologique que la voie cartésienne sont indirectes dans la mesure où elles rejoignent la sphère transcendantale uniquement par une critique de l'expérience mondaine (p. 33-34 ; voir 8/293). La voie cartésienne le fait par l'entremise d'une exposition de l'imperfection de l'expérience mondaine. Comparer I. Kem, « Die drei Wege zur transzendental-phänomenologischen Reduktion in der Philosophie Edmund Husserls ». Tijdschrift voor Filosofie 24, 1962, p. 303-349 et J. J. Drummond, « Husserl on the Ways to the Performance of the Reduction », Man and World 8, 1975, p. 47-69. Ce dernier soutient de façon convaincante que la supposée voie psychologique vers la réduction est en réalité un cas spécial de la voie ontologique (p. 64).

7. Quoique j'insiste sur l'aspect direction-sur-le-monde dans mon élucidation du motif régissant le projet phénoménologico-transcendantal, il est important toutefois de ne pas négliger l'aspect éthique. La recherche de Husserl d'un fondement apodictique est solidement relièe à son idée d'une auto-responsabilité absolue (8/ 197). Il parle d'une vie auto-responsable ultime en pleine clarté et transparence, rendue possible par l'auto-examen radical prenant place dans la recherche d'un fondement transcendantal $(8 / 167)$. Ainsi, vivre sa vie dans l'attitude phénoménologique conduira éventuellement à une transformation personnelle d'une signification existentielle vitale (6/140). En d'autres mots, la philosophie est étroitement liée à la pratique lpraxisl éthique et. dans Erste Philosophic I, il réfère explicitement et rend hommage à l'idée socratique-platonicienne de la philosophie $(7 / 9)$. Cette impulsion éthico-normative est d'une importance particulière lorsque la tentative d'atteindre un fondement apodictique pour la philosophie scientifique se révèle être un idéal infini. Précisément. la quête pour l'auto-responsabilité absolue peut nous pousser de l'avant dans une critique radicale perpétuelle, à la recherche de l'évidence absolue (8/196, 8/244, 5/139, I/53). 
La relation mentionnée plus haut entre la subjectivité comme sujet de l'expérience et la subjectivité comme constituante peut être clarifiée par une brève introduction à la théorie husserlienne de l'intentionnalité. Bien plus, il s'agit là du cadre auquel appartient originairement le concept de constitution. Une des visées centrales des Recherches logiques est de montrer combien trompeuse peut être cette façon de parler de notre expérience d'un objet comme si l'objet entrait, en quelque sorte, dans la conscience. De plus, il est faux de dire que la conscience comme sujet de l'expérience entre en relation avec les objets ou d'affirmer que l'acte intentionnel contient en lui-même quelque chose comme son objet ( $\mathrm{g} / 3 / 355)$. Une analyse phénoménologique de la structure de l'expérience révèle que la « relation » intentionnelle entre l'acte et l'objet est précisément intentionnelle, non réelle, et que la directionalité est un trait non relationnel intrinsèque à l'acte intentionnel, et n'est pas quelque chose qui apparaît et disparaît dépendamment de l'existence de l'objet intentionnel. Conséquemment, la directionalité d'un acte vers l'objet n'implique pas que deux entités séparées soient données - l'objet et l'acte :

ICl'est une seule chose qui est présente, le vécu intentionnel, dont le caractère descriptif essentiel est précisément l'intention relative à l'objet. C'est cette intention qui, selon sa spécification particulière, constitue totalement et à elle seule la représentation ou le jugement de cet objet, etc. ( $19 / 386)$.

Qu'une représentation se rapporte à un certain objet, et cela d'une certaine manière, elle ne le doit assurément pas à une opération qu'elle exercerait sur l'objet existant en soi, hors d'elle, comme si elle se « dirigeait » vers lui au sens littéral du mot, ou comme si, de quelque autre manière, elle s'occupait de lui et le manipulait, par exemple, comme la main qui écrit a affaire à la plume ; ce rapport à l'objet, elle ne le doit en aucune façon à quelque chose qui demeure de quelque manière extérieur à elle, mais exclusivement à son caractère propre ( $\mathrm{g} / 45 \mathrm{I}$ ).

D̛un point de vue phénoménologique, il y a seulement l'acte et son corrélat intentionnel objectif : « Être objet n'est pas un caractère positif, ni une espèce particulière de contenu, cela désigne seulement le contenu comme corrélat intentionnel d'une représentation » $(\mathrm{ig} / 6 \mathrm{i} 6)$. Dorénavant, les analyses de Husserl n'impliquent pas seulement que nos actes intentionnels sont caractérisés par la directionalité. Toutes les variations de la description de notre orientation-sur-l'objet (incluant la description de l'objet qu'elle comporte nécessairement) doivent être expliquées par, et fondées dans, l'expérience intentionnelle elle-même 
$(19 / 427,3 / 74)$. Ceci n'implique pas, toutefois, que la relation est immanente - ce qui constituait précisément la revendication erronée de Brentano ${ }^{8}$. L'acte, en lui-même, ne contient pas l'objet de façon immanente (19/385). Il ne s'agit pas de l'emboîtement d'un contenu psychique dans un autre. Car, comme Husserl l'a démontré en maintes occasions, il y a une nette distinction entre la donation du processus mental temporel, en tant que contenu immanent, et l'objet intentionnel, et ce dernier - et ceci est également vrai d'un objet imaginé, non-existant - est transcendant à l'acte 9 .

Ces brèves considérations peuvent nous aider à élucider le rapport entre intentionnalité et constitution. Car, comme le dit Husserl, l'analyse de l'intentionnalité nous conduit à l'aperçu suivant lequel

(...) les objets dont nous devenons " conscients », ne sont pas simplement présents dans la conscience comme dans une boîte, de telle sorte qu'on n'ait qu'à les y trouver et à les saisir ; mais que c'est dans des formes diverses de l'intention objective qu'ils se constituent comme ce qu'ils sont et valent pour nous ( $19 / 169$. Voir aussi $\left.2 / 7 \mathrm{I}^{-7} 75\right)$.

Ainsi, l'intentionnalité implique, dans sa prestation objectivante et identifiante, une construction de l'identité objective. C'est-àdire que l'identité de l'objet de connaissance - l'identité de l'objet intentionnel - a comme prérequis l'accomplissement d'un acte. Lorsque nous percevons un encrier par exemple, cela signifie simplement, d'un point de vue phénoménologique, qu'une certaine somme de sensations sont imprégnées d'un certain caractère d'acte, un acte d'appréhension objectivant et homogène. Ce qui veut dire que l'apparaître de l'objet intentionnel - sa constitution - est le résultat d'une interaction entre la sensation et l'aperception. Lorsque des sensations sont sujettes à une interprétation objectivante, elles forment l'objet intentionnel ${ }^{\mathrm{IO}}$.

8. Voir Brentano, Psychologie vom empirischen Standpunkt I, Hamburg. Felix Meiner, 1971, p. 125 .

9. On retrouvera une présentation plus détaillée dans D. Zahavi, « Intentionality and the Representative Theory of Perception », Man and World (à paraitre), 1993 .

10. Un examen minutieux du concept husserlien de sensation [Empfindungl et une description de la façon dont il a été graduellement purifié de son penchant sensualiste initial peuvent être trouvés dans R. Sokolowski, The Formation of Husserl's Concept of Constitution, La Haye, Martinus Nijhoff, 1970 et E. Holenstein, Phänomenologie der Assoziation, La Haye, Martinus Nijhoff, 1972. 
L'aperception est, pour nous, ce surplus qui consiste dans le vécu lui-même, dans son contenu descriptif, par opposition à l'existence brute de la sensation; c'est le caractère d'acte qui anime pour ainsi dire la sensation et qui, selon son essence, fait en sorte que nous percevons tel ou tel objet, que, par exemple, nous voyons cet arbre, nous entendons cette sonnerie, nous sentons le parfum de telle fleur, etc. (19/399).

La question qui demeure est de savoir comment on doit interpréter ce processus. Est-ce l'objet réel dans son identité propre qui est constitué, ou est-ce simplement l'objet phénoménal, c'est-à-dire l'objet en tant qu'objet de connaissance ? Nous sommes, en d'autres mots, confrontés au dilemme classique : la constitution doit-elle être comprise comme une restitution ou comme une production, ou éventuellement comme quelque chose de tout à fait différent? Une question à laquelle je tenterai de répondre plus tard.

Revenant à notre première discussion, nous pouvons conclure que les sciences mondaines étudient seulement les objets constitués - sans jamais les thématiser en tant que constitués - alors que la phénoménologie transcendantale analyse la corrélation entre les objets constitués et la subjectivité constituante, découvrant ainsi la dépendance du sens d'être véritable de la réalité objective $(6 / 179,8 / 457,9 / 336$, g/19I). Alors que l'attitude naturelle est caractérisée par une préoccupation ontique et directe avec les objectivités, qui dissimule leur caractère constitué, c'està-dire leur être en tant qu'unités intentionnelles de validité $(g /$ 344), la réduction transcendantale découvre la subjectivité performante et l'a priori de la corrélation.

Il est par conséquent possible de résumer notre présentation de la manière suivante. Le but de l'épochè et de la réduction n'est pas d'abandonner le monde ou de changer son sens propre ${ }^{\mathrm{II}}$. Au contraire, leur tâche est de nous ouvrir une voie d'accès au monde de telle sorte qu'elle nous permette de découvrir son sens véritable (8/457). C'est-à-dire que l'époché et la réduction sont accomplies

II. Occasionnellement, Husserl décrit l'épochè comme la condition de possibilité de la réduction (6/154), et quoiqu'elles fassent toutes deux partie d'une unité fonctionnelle, elles doivent tout de même être différenciees. Alors que l'épochè est une suspension du jugement ontique naîf et donc peut être caractérisée comme la porte d'entrée [Eingangstor (6/26o). la réduction est le changement d'attitude qui thématise la corrélation entre le monde et la conscience, découvrant ultimement le fondement transcendantal (I/6I). 
dans le but de rendre l'être du monde compréhensible ${ }^{\mathrm{I} 2}$, et Husserl met explicitement l'emphase sur le fait que l'être du monde n'est en aucune façon exclu de la sphère de recherche phénoménologique (8/432). La prétendue exclusion du monde est en réalité une exclusion d'une conception présupposée du monde :

La réalité empirique n'est ni « dénaturée » [umgedeutet], ni même niée, mais nous écartons une interprétation absurde qui contredit son sens propre tel qu'il est élucidé par l'évidence (3/120; voir 8/465).

A la lumière de cette interprétation, il est clair que l'épochè n'implique pas une perte. Au contraire, le changement d'attitude rend possible une découverte fondamentale qui, ultimement, élargit notre sphère d'expérience $(6 / 154, \mathrm{I} / 66)$. Husserl compare la pratique de l'épochè à la transition d'une vie à deux dimensions à une vie à trois dimensions (6/120). Soudainement, la subjectivité transcendantale, qui jusqu'alors fonctionnait tout en demeurant voilée, se révèle comme la dimension indispensable de la donation et de la découverte du sens.

« Le » monde n'est pas perdu par l'époché, et celle-ci n'est absolument pas une abstention eu égard à l'être du monde et de chaque jugement sur lui, mais elle est le chemin du dévoilement des jugements de corrélation, de la réduction de toutes les unités d'être à moi-même et à ma subjectivité, qui possède le sens et en est donatrice, avec toutes ses potentialités ( $15 / 366$. Nous soulignons).

Il est maintenant possible d'expliquer pourquoi le projet de Husserl doit être caractérisé comme un projet phénoménologicotranscendantal. Il est transcendantal dans la mesure où l'explicitation du sens d'être du monde révèle sa dépendance à l'égard de la conscience, la subjectivité étant la condition de possibilité de toute donation. Ainsi, Husserl parle de la réduction transcendantale parce que la phénoménologie nous conduit de la sphère naturelle de l'être dérivé à son fondement transcendantal (I/6I ; voir $3 /$ 159). Le projet de Husserl est également phénoménologique dans son approche. Contrairement aux questions de Kant - comment les jugements synthétiques a priori sont-ils possibles ? Quelles sont les conditions de possibilité de l'expérience objective? -, les questions de Husserl sont régies par le principe phénoménologique. Uniquement par la voie d'une élucidation du sens d'être du

12. Voir les remarques de Husserl dans E. Fink. VI. Cartesianische Meditation II, Dordrecht, Kluwer Academic Publishers, 1988, p. 78. 
monde sommes-nous conduits à la découverte transcendantale de la subjectivité constituante ( $\mathrm{I} / \mathrm{I} 77$ ).

Cette interprétation nous permet de confronter les deux mésinterprétations mentionnées plus haut concernant le but et l'objet de la phénoménologie. Il a déjà été démontré que l'époché et la réduction n'impliquent aucune exclusion du monde. Toutefois, il est aussi nécessaire de critiquer la vue selon laquelle les prétentions de la phénoménologie husserlienne sont exclusivement théorético-sémantiques ${ }^{\mathrm{I} 3}$. La réduction phénoménologique n'exige pas la neutralité sur les questions existentielles pas plus que la phénoménologie husserlienne ne peut être associée à une sorte d'analyse conceptuelle similaire aux formes récentes de la philosophie anglo-américaine ${ }^{14}$. Lorsque les analyses phénoménologiques portent sur le sens du monde et décrivent sa constitution, il ne s'agit pas simplement de l'explicitation d'une pure sphère du sens, séparée du monde réel existant. Ce n'est pas comme si l'analyse phénoménologique pouvait ou devait être complétée d'une analyse ontologique de l'être. Husserl n'est pas préoccupé par des réflexions théorético-sémantiques qui sont dénuées d'implications métaphysiques et ontologiques, et décrire les réflexions de Husserl de cette façon, ce serait négliger leur caractère philosophico-transcendantal qui, dans sa nature même ${ }^{15}$, remplace la distinction objectiviste entre sens et être ${ }^{16}$. Ainsi, Husserl admet volontiers qu'une phénoménologie

13. Parmi les défenseurs de cette approche, on peut mentionner R. H. Holmes dans «Is Transcendantal Phenomenology Committed to Idealism ? », Monist 59, 1975. p. 98-I14 ; H. Hall, « Was Husserl a Realist or an Idealist? » dans H. Dreyfus (dir.), Husserl, Intentionality and Cognitive Science, Cambridge Mass., M.I.T. Press, 1982, p. 169190 ; et P. Hutcheson, « Husserl's Problem of Intersubjectivity », Joumal of British Society of Phenomenology 11, 1980, P. 144-162 et « Solipsistic and Intersubjective Phenomenology », Human Studies 4, 1981, p. 165-178.

14. Voir P. Hutcheson, ibid., p. 168.

15. Conséquemment, la tentation d'endosser l'interprétation théorético-sémantique en faisant référence aux passages où Husserl parle de la constitution du sens est inutile puisque Husserl a dépassé la compréhension objectiviste de ce terme (comme quelque chose différent de l'être) par sa pratique de la réduction. Ceci n'implique pas, évidemment, que chaque entité signifiante existe. Lorsque nous parlons d'un objet existant, nous parlons d'un objet dans un mode de donation prééminent, un objet présent intuitivement, ou pour être plus précis : d'une intention étant intuitivement remplie. Cest-à-dire que seulement un certain type de sens, le donné perceptuel (c'est-à-dire le sens perceptuel au sens large), nous permet de parler de l'existence de l'objet. Voir D. Zahavi, Intentionalität und Konstitution. op.cit., p. 109 .

16. Cette distinction serait acceptable uniquement dans la phénoménologie pré- 
transcendantale pleinement développée est eo ipso la véritable et réelle ontologie ( $1 / 138,8 / 215)$, alors que tous les concepts ontologiques sont élucidés dans leur corrélation avec la subjectivité constituante. L'élucidation et la clarification transcendantales ainsi accomplies peuvent être caractérisées comme l'explication philosophique ultime ( $\left./ / \mathrm{I}_{1} 8\right)$, et Husserl soutient constamment que seule une explication transcendantale peut conduire à la véritable intelligibilité (6/193):

Reconduire aux origines intentionnelles, aux unités intentionnelles de la formation du sens, cela produit une intelligibilité, laquelle une fois atteinte (mais c'est là, il est vrai, un cas idéal) ne laisserait derrière elle aucune question pourvue de sens (6/I7I).

De plus, Husserl est très explicite dans son rejet de la mésinterprétation antimétaphysique :

Finalement, afin d'éviter toute mécompréhension, je voudrais faire remarquer que seule cette métaphysique naïve opérant avec des choses en soi contradictoires est exclue par la phénoménologie mais pas la métaphysique en général. L'être premier en soi, celui qui prime sur toute objectivité mondaine et les porte est l'intersubjectivité transcendantale, l'ensemble des monades qui se communitarisent dans diverses formes ( $\mathrm{I} / 3^{8}-9$ ).

La phénoménologie est anti-métaphysique dans la mesure où elle récuse toute métaphysique se mouvant dans des substructions formelles et vides. Mais tout problème philosophique authentique, tous les problèmes métaphysiques sont ramenés sur le sol phénoménologique où ils trouvent leur véritable méthode et configuration transcendantales puisées de l'intuition $(g / 253)$.

transcendantale de Husserl. Ainsi, dans les Recherches logiques, Husserl rejette comme questions métaphysiques n'ayant de place en phénoménologie, les questions concernant l'existence et la nature du monde extérieur ( $\mathrm{i} / 26)$. De plus, il affirme qu'il est sans importance, pour une description phénoménologique, qu'une perception soit véridique ou trompeuse puisque la tâche de la phénoménologie (descriptive) consiste précisément dans la description du phénomène en tant que pur phènomène. Aussi longtemps que toutes les questions concernant la réalité objective de l'objet intentionnel furent suspendues, la phénoménologie de Husserl fut toutefois confrontée à de graves insuffisances - un fait que Husserl, plus tard, admit fréquemment (voir à ce propos Husserl, « Entwurf einer "Vorrede" zu den "Logischen Untersuchungen" (1913) 》. Tijdschrift voor Filosofie I, (1939), p. nio et 117. Voir aussi l'exposé détaillé dans D. Zahavi, Intentionalität und Konstitution, op. cit. La compréhension du champ, de l'objet et des prétentions de la phénoménologie changea toutefois au moment où Husserl passa à la phénoménologie transcendantale, et dans la dernière partie des Idées I - intitulée raison et réalité - le problème de la réalité objective et sa corrélation avec la conscience rationnelle [Vernunftbewußtsein] est explicitement traitée (voir à ce propos 3/313). C'est ce traitement qui, ultimement, conduisit Husserl vers une conception de l'intersubjectivité transcendantale comme fondement de l'objectivité et de la réalité du monde. 
Comme le dit Landgrebe, la réduction est la voie de Husserl vers le cœur des problèmes métaphysiques ${ }^{\mathrm{I} 7}$.

\section{III}

La pratique de l'épochè et de la réduction transcendantale mène à une compréhension du rôle joué par la subjectivité. Tout être transcendant, c'est-à-dire tout objet avec un mode de donation horizontal, doit nécessairement - si on veut éviter toute présupposition dogmatique - être en corrélation avec une subjectivité qui en fait l'expérience (une subjectivité constituante), et doit dorénavant être examiné uniquement selon cette corrélation. Conséquemment, toute assertion à propos d'une transcendance absolument indépendante de l'esprit est inacceptable, et nous sommes donc confrontés au problème de l'idéalisme husserlien.

Dans la préface à la deuxième Recherche logique, Husserl écrit que seule une épistémologie idéaliste est cohérente (Ig/II2). A cette époque, toutefois, l'idéalisme signifiait simplement une théorie dëfendant l'irréductibilité des objets idéaux. Dans les Méditations cartésiennes, on retrouve la défense de la même thèse (I/) II8) quoique le sens en soit maintenant radicalement changé. Après le tournant transcendantal, l'idéalisme est compris comme une théorie défendant la suprématie ontologique de la subjectivité (8/215). Une suprématie que Husserl semble considérer comme le cour du débat phénoménologique :

Celui qui comprend mal le sens profond de la méthode intentionnelle ou le sens de la réduction transcendantale - ou l'un et l'autre - peut seul vouloir séparer la phénoménologie et l'idéalisme transcendantal ( $\mathrm{I} / \mathrm{Irg}$ ).

Quoique Husserl caractérise la phénoménologie comme la première version strictement scientifique de l'idéalisme transcendantal (8/18I), le problème majeur est toutefois de découvrir le caractère distinct et unique de cet idéalisme. Car Husserl souligne sans cesse le fait que l'idéalisme phénoménologico-transcendantal est radicalement différent de l'idéalisme traditionnel qui, dans son opposition traditionnelle au réalisme, manifeste simplement sa réclusion dans l'attitude naturelle (5/149-153, I7/178, I/33-4, I/

17. Cf. L. Landgrebe, Der Weg der Phänomenologie. Das Problem der ursprünglichen Erfahrung. Gütersloh, I963. P. 26. 
II8). Ainsi, Husserl ne soutient pas que l'objet intentionnel doit être compris comme un complexe de sensations immanentes ( $\mathrm{ig}$ / 764), pas plus qu'il ne questionne ou ne doute de l'existence actuelle du monde réel. La thèse idéaliste n'implique aucune dissolution de la transcendance mondaine dans l'immanence du psychique (3/335), pas plus qu'elle ne nie l'existence du monde. Bien au contraire, la tâche de l'idéalisme transcendantal - phénoménologique - est d'en arriver à une compréhension philosophique de la transcendance mondaine par la mise à découvert systématique de l'intentionnalité constituante (I/34). L'idéalisme transcendantal est ultimement une interprétation du sens préphilosophique du monde ( $1 / 36$ ), et Husserl, conséquemment, affirme que ce type d'idéalisme comprend en lui le réalisme naturel $(g / 254)$ :

Que le monde existe, qu'il soit donné comme Universum existant dans l'expérience continue s'accordant toujours universellement, cela est absolument indubitable. Il en va tout autrement lorsqu'il s'agit de comprendre l'indubitabilité eu égard à la vie et à la science positive, et d'en élucider le fondement ( $5 / 153)$.

Il ne peut donc y avoir de réalisme plus fort, si par ce mot on n'entend rien de plus que : «je suis certain d'être un homme, qui vit dans ce monde, etc., et je n'ai pas là-dessus le moindre doute ». Mais c'est justement le grand problème, que de comprendre cette « évidence » $(6 / 191)^{\mathrm{I}}$.

Bien que Husserl défende apparemment la thèse de la dépendance du monde constitué vis-à-vis de la subjectivité constituante (3/104-6, 3/159, 5/153), il est important de noter la transformation que ces concepts ont subi suite à la réduction. Husserl fait souvent remarquer que c'est une mécompréhension décisive que d'interpréter la corrélation dans le cadre de l'opposition traditionnelle sujet / objet (6/265). Être et conscience sont essentiellement interdépendants, et font ultimement un dans la concrétion absolue : la subjectivité transcendantale $(\mathrm{I} / \mathrm{I} / 7)$. Cest-à-dire que la notion husserlienne de "subjectivité » est éventuellement élargie de telle sorte qu'elle dépasse et peut-être même mine non seulement l'opposition traditionnelle sujet-objet mais aussi la vue qui tend à concevoir leur relation comme une stricte (et statique) corrélation.

18. Voir aussi la lettre de Husserl à l'Abbé Baudin citée dans I. Kern, Husserl und Kant. Eine Untersuchung über Husserls Verhältnis zu Kant und zum Neukantianismus, La Haye, Martinus Nijhoff, 1964, p. 276. 
Ces remarques peuvent servir comme une indication du caractère complexe de l'idéalisme husserlien. Dans son article Die phänomenologische Bewegung, Gadamer rend hommage à Husserl pour son dépassement du vieil antagonisme pré-critique entre réalisme et idéalisme ${ }^{\mathrm{I} 9}$. Une interprétation que j'endosse et que je vais essayer de renforcer en examinant quelques remarques tardives de Husserl sur la constitution.

IV

Une approche traditionnelle du problème des implications ontologiques du concept de constitution, c'est-à-dire l'aspect qui porte directement sur la controverse réalisme / idéalisme, a été d'utiliser la vieille opposition entre ontologie et épistémologie. Ainsi, le concept - transcendantal - husserlien de constitution a été notamment interprété comme une restitution (réaliste) ou une production / création (idéaliste) de l'objet intentionnel. Une question urgente, toutefois, est de savoir si ces alternatives sont viables lorsqu'on en vient à la compréhension véritable de la constitution phénoménologico-transcendantale. Ne demeurent-elles pas - dans leur acceptation de la distinction stricte entre l'ordre de l'être et l'ordre de la connaissance - situées dans un objectivisme qui est dépassé par la pratique de la réduction? Peut-être la critique traditionnelle de Husserl, n'ayant jamais donné de réponse décisive à la question de savoir si la constitution est une restitution ou une production, est-elle injustifiée, posant la mauvaise question dès le départ ${ }^{20}$ ?

En dépit de cette remontrance, je suis loin de dénier que le concept husserlien de constitution est caractérisé par une imprécision essentielle, et ultimement, la tâche de clarification de ce concept-clé repose sur l'interprète puisque Husserl lui-même ne

19. H. G. Gadamer, " Die phänomenologische Bewegung », Kleine Schriften III. Tübingen, J. С. В. Mohr, 1972, p. I78. Voir les réflexions de E. Fink, V. Cartesianische Meditation I, Dordrecht, Kluwer Academic Publishers, 1988, p. 170-179, qui se terminent par la conclusion suivante : « Die Einsicht in diese Zusammenhänge läßt eindeutig erkennen, wie der transzendentale Idealismus jenseits von Idealismus und Realismus ist. »

20. Cest, à ce propos, plutôt étonnant que les tenants de l'interprétation créationniste, qui font souvent référence au fameux article de Fink « Die phänomenologische Philosophie Edmund Husserl in der gegenwärtigen Kritik », Kantstudien 38 , 1933. P. 319-383, ne semblent jamais avoir porté assez attention à sa formulation exacte. Fink parle du caractère productif de l'intentionnalité transcendantale, et écrit que l'essence de la constitution doit être définie comme une 
s'est jamais chargé de sa thématisation et de son élaboration explicites.

Un principe central dans les dernières considérations de Husserl est le postulat selon lequel l'activité constituante est caractérisée par une sorte de réciprocité dans la mesure où l'agent constituant est lui-même constitué par le processus de constitution (voir à ce propos 15/546). Ce point est d'une importance capitale puisqu'il remet encore une fois en question l'idée d'un sujet isolé, détaché du monde.

Nous avons vu que Husserl affirmait, dans les Méditations Cartésiennes, que l'être et la conscience sont essentiellement interdependants et font ultimement un dans la concrétion absolue : la subjectivité transcendantale ( $\mathrm{I} / \mathrm{I} \mathrm{I} 7)$. Conséquemment, la « monade », comme terme de la subjectivité transcendantale dans sa pleine concrétion, enveloppe non seulement le pôle égologique dans le flux de son vécu intentionnel, mais également les objets transcendants visés et constitués ( $\mathrm{I} / 26, \mathrm{I} / \mathrm{IO} 2, \mathrm{I} / \mathrm{1} 35, \mathrm{I} 4 / 46)$. Cet èlargissement du concept traditionnel de subjectivité conduit ultimement à une reconsidération décisive de la relation entre la subjectivité transcendantale et le monde. Une reconsidération qui rompt avec l'ancienne conception statique de leur corrélation. Ainsi, Husserl note que le monde peut être conçu comme la mondaneisation transcendantale de l'intersubjectivité transcendantale $(15 / 403)$ ! Fink préconise une approche similaire qui affirme que le véritable thème de la phenomènologie n'est ni le monde, ni une subjectivité sans monde, mais le devenir du monde dans l'(auto-)constitution de la subjectivité transcendantale ${ }^{2 \mathrm{I}}$. Ainsi, aucune corrélation réellement constitutive n'existe entre la subjectivité transcendantale et le monde. Bien plutôt, l'activité constituante doit être vue comme l'auto-réalisation de la subjectivité constituante dans la réalisation du monde ${ }^{22}$. On peut retrouver des affirmations similaires dans Ideen III, où Husserl, en rapport avec la description de la constitution du corps, écrit : « Ce qui doit

création productive (p. 373). Dans la même phrase, cependant, il poursuit en déclarant que cette caractérisation est faite principalement dans le but d'éviter toute interprétation réceptive, ontico-mondaine, de la constitution et il conclut en soutenant que la constitution n'est, ultimement, ni un processus réceptif, ni un processus productif mais quelque chose qui ne peut être saisi par des concepts ontiques (p. 373).

2I. E. Fink, ibid., p. 370.

22. E. Fink, VI. Cartesianische Meditation II, op. cit., p. 49. 
plus particulièrement être pris en compte, c'est le rapport mutuel constant de la constitution du moi et du corps, d'une part, et de la choséité réelle, d'autre part $[. . .1 \gg(5 / 128$; voir $16 / 162)$.

Pour un compte rendu plus détaillé de ces questions, on doit cependant tenir compte de la théorie husserlienne de l'intersubjectivité transcendantale - et plus spécifiquement sa description du processus de monadisation. Je ne peux fournir ici une analyse de cet aspect très important de la phénoménologie de Husserl, mais je me permets de mentionner que Husserl considère que la mise en place de l'intersubjectivité transcendantale - qu'il conçoit comme le fondement transcendantal de l'objectivité et de la mondanité - doit aller de pair avec une auto-objectivation réciproque des sujets transcendantaux $(8 / 505)^{23}$. Une auto-objectivation qui n'est pas antérieure ou subséquente à la constitution du monde, mais qui en est contemporaine ( $\mathrm{I} / \mathrm{r} 30$ ). Ainsi, l'autodéploiement et la mondanéisation du sujet transcendantal, la mise en place de l'intersubjectivité transcendantale et la possession-du-monde [Welthabel font partie d'un processus simultané et interrelié :

Moi, l'ego, je possède donc le monde par une prestation, dans laquelle d'un côté je me constitue, avec mon horizon d'autrui, et constitue du même coup la communauté homogène des « Nous », et cette constitution n'est pas celle du monde, mais bien une prestation qui peut être décrite comme la monadisation de l'ego - comme la prestation de la monadisation personnelle, de la pluralisation monadique $66 / 416-7$ de Mai 1937 ; voir $15 / 639,15 / 368)$.

A première vue, cette révocation de la différence entre sujet et monde semble impliquer un accroissement décisif de l'autorité de l'(inter)subjectivité transcendantale. En fait, la conséquence est à l'opposé direct, et ceci peut jeter un nouvel éclairage sur le concept de constitution.

Parler, de façon formelle, de la constitution comme étant la condition subjective de l'apparition de l'objet, comme une façon de laisser l'objet se donner [zur Gegebenheit bringen des Gegenstandes] $(14 / 47)^{24}$ ne signifie pas une simple reproduction épistémique de quelque chose déjà existant, pas plus que cela n'implique une

23. Voir Manuscrit $C_{1} 733 a, 15 / 373$ et $13 / 480$.

24. Une description que Heidegger réitère : “Konstituieren" meint nicht Herstellen als Machen und Verfertigen, sondern Sehenlassen des Seienden in seiner Gegenständlichkeit. », Prolegomena zur Geschichte des Zeitbegriffs, Gesamtausgabe Band 2o, Frankfurt, V. Klostermann, 1979, P. 97. 
réceptivité au sens où le processus constitutif serait influencé par un stimulus externe - qu'il s'agisse d'une chose en soi inconnaissable ou d'entités physiques. Comme Husserl l'a déjà mentionné dans les Recherches logiques : les concepts dedans et dehors ont leur origine dans la métaphysique naïve de la vie quotidienne (ig/673, I $9 / 708$ ). D'un autre côté, la constitution ne manifeste pas simplement une production ex nihilo arbitraire du sujet transcendantal, comme si elle était délibérément dirigée et guidée par lui ${ }^{25}$. Dans un manuscrit de i93r, Husserl écrit :

La constitution des étants de couches différentes, des mondes, des temps, a deux présuppositions essentielles, deux sources originaires qui, temporellement parlant (dans chacune de ces temporalités), les sous tendent toujours : I) mon moi premier comme moi originaire en fonction de ses affections et actions, et avec toutes les configurations d'essence appartenant à ces modes ; 2) mon non-moi premier comme flux originaire de temporalisation et, en tant que tel, comme forme originaire de la temporalisation, un champ temporel constituant l'originarité. Cependant, les deux origines sont unies, inséparables et, considérées pour elles-mêmes, abstraites ${ }^{26}$.

Il poursuit en parlant du non-ego fluant originaire qui constitue l'univers hylétique - c'est-à-dire le champ de donation primordiale sur lequel sont basées nos interprétations intentionnelles indépendamment de toute participation de l'ego, quoique l'ego soit toujours présent (dabeil (Manuscrit $\mathrm{C}$ io I6a). Les deux fondements sont, comme le dit Husserl, inséparables. Ainsi, Husserl n'entend d'aucune façon déduire le monde, la hylè ou l'a priori ontique de l'ego ${ }^{27}$. La raison, toutefois, pour laquelle ils doivent toujours être compris en corrélation avec l'ego - pour laquelle ils doivent toujours être considérés comme subjectifs - est que la subjectivité transcendantale demeure le lieu où ils apparaissent, c'est-à-dire où ils peuvent se manifester et se déployer (voir I5/

25. La constitution des objets culturels est évidemment une exception mais ils sont, à cet égard, de moindre importance.

26. «Konstitution von Seienden verschiedener Stufen, von Welten, von Zeiten hat zwei Urvoraussetzungen, zwei Urquellen, die zeitlich gesprochen "in jeder dieser Zeitlichkeiten" immerfort ihr "zugrundeliegen": I) mein urtümliches Ich als fungierendes Ur-Ich in seinen Affektionen und Aktionen, mit allen Wesensgestalten an zugehörigen Modis; 2 ) mein urtürnliches Nicht-Ich als urtümlicher Strom der Zeitigung und selbst als Urform der Zeitigung, ein Zeitfeld, das der UrSachlichkeit, konstituierend. Aber beide Urgründe sind einig, untrennbar und, so für sich betrachtet, abstrakt » (Manuscrit C 10 $15 b$. Nous soulignons).

27. Cette dernière interpretation a déjà été soutenue par $E$. Tugendhat, Der Wahrheitsbegriff bei Husserl und Heidegger, Berlin, Walter de Gruyter \& Co., 1970, p. 177, 212, 217 et Sokolowski, op.cit., p. 138, 159, 197-198, 217. 
434). Si cette interprétation est acceptée, elle devrait aussi clarifier la notion husserlienne concernant la fonction donatrice de sens de l'(inter)subjectivité transcendantale (8/457, 17/25I, 15/366). Parler de la subjectivité comme étant donatrice de sens, c'est parler de la subjectivité comme étant le lieu où le sens de l'objet peut se déployer et se manifester. Cette donation de sens ne doit pas être comprise comme une animation arbitraire de sensations dénuées de sens par une subjectivité autonome.

En même temps, il devrait être clair que nous n'avons plus désormais affaire à une subjectivité transcendantale qui peut s'abstenir de s'engager dans l'activité constituante, si elle le désire, pas plus qu'elle n'est séparée et détachée du processus constitutif. Au contraire, la constitution manifeste l'auto-réalisation de l'(inter)subjectivité constituante. C'est-à-dire que l'agent transcendantal est lui-même constitué dans le processus de constitution, c'est-à-dire, dans le fait de laisser le monde lui-même se manifester. Ainsi, la constitution transcendantale est un processus prenant place et se déployant dans la structure monde/ conscience ${ }^{28}$.

Par cette interprétation, il devrait être clair pourquoi l'idéalisme transcendantal de Husserl a dépassé les concepts traditionnels de réalisme et d'idéalisme. A tout le moins si le réalisme est compris comme une théorie qui défend l'existence d'entités indépendantes de l'esprit, c'est-à-dire qui affirme que l'être de la nature, du monde et des entités mondaines sont sans aucune référence à la subjectivité qui en fait l'expérience ${ }^{29}$; et si l'idéalisme est compris comme une théorie qui défend la suprématie de la conscience de telle sorte que, soit elle conçoit tous les objets comme étant des parties immanentes de la conscience, soit elle considère la conscience sans monde comme source unique du sens d'être du monde.

Il devrait aussi être clair, toutefois, que les affinités entre les vues de Husserl sur la relation entre subjectivité et monde, et celles des par les derniers phénoménologues sont beaucoup plus

28. Husserl parle occasionnellement d'une Weltbeur ßtseinsleben (par exemple dans le manuscrit KIII6 de 1936 qui vient de paraître dans le volume 29 des Husserlianal, et bien que je ne puisse le démontrer ici, on doit réaliser que le processus constitutif, ultimement, se déploie lui-même dans une structure tripartite incluant la subjectivité, l'intersubjectivité et le monde. Comme il a dejjà étē mentionné dans Ideen II : je, nous et le monde appartiennent l'un à l'autre (4/288).

29. Voir à ce propos la définition de N. Rescher, Human Knowledge in Idealistic Perspective, Princeton, Princeton University Press, 1992, p. 255. 


\title{
grandes que les interprétations décrivant Husserl comme un crypto-cartésien ne sont prêtes à l'admettre ${ }^{30}$.
}

\author{
Université de Copenhague
}

(Tradutt de l'anglais par Daniel Cayer)

30. Je pense ici à l'analyse générale de Heidegger de la structure du Dasein, l'être-aumonde. Par exemple, lorsqu'il écrit : «Im Sichverstehen ist das In-der-Welt-Sein verstanden, womit bestimmte Möglichkeiten des Mitseins mit Anderen und des Umgangs mit innerweltlichem Seienden vorgezeichnet sind.Im Sichverstehen als In-der-Welt-Seinkönnen ist gleichursprünglich Welt verstanden » (Heidegger, Grundprobleme, op. cit., P.394). « Welt existiert, dh. sie ist nur, sofem Dasein da ist Nur wenn Welt da ist, wern Dasein als In-der Weltsein existiert, ist Seinsverständnis da, und nur wenn dieses existiert, ist innenweltliches Seiendes als Vorhandenes und Zuhandenes enthüllt Weltverständnis als Daseinsuerständnis ist Selbstverständnis. Selbst und Welt gehören in dem einen Seienden, dem Dasein, zusammen. Selbst und Welt sind nicht zwei Seiende, wie Subjekt und Objekt, auch nicht wie Ich und Du, sondern Selbst und Welt sind in der Einheit der Struktur des In-der-Welt-Seins die Grundbestimmung des Daseins selbst. » (Ibid., p.422 ; voir les commentaires de Tugendhat dans op. cit, p.263). De même, le passage suivant de la Phénoménologie de la perception de Merleau-Ponty révèle quelques parallèles frappants : « Le monde est inséparable du sujet, mais d'un sujet qui n'est rien que projet du monde, et le sujet est inséparable du monde, mais d'un monde quil projette lui-même. Le sujet est être-au-monde et le monde reste subjectif puisque sa texture et ses articulations sont dessinées par le mouvement de transcendance du sujet. Nous découvrions donc avec le monde comme berceau des significations, sens de tous les sens, et sol de toutes les pensées, le moyen de dépasser l'altemative du réalisme et de lidéalisme, du hasard et de la raison absolue, du non-sens et du sens. ", Paris, Gallimard, 1945, p. $49 \mathbf{1}^{-2}$. 